\title{
Safety and Feasibility of Percutaneous Dilatational Tracheostomy Performed by Intensive Care Trainee
}

Daesang Lee, M.D., Chi Ryang Chung, M.D., Sung Bum Park, M.D., Jeong-Am Ryu, M.D., Joongbum Cho, M.D., Jeong Hoon Yang, M.D., Chi-Min Park, M.D., Gee Young Suh, M.D., and Kyeongman Jeon, M.D.

Department of Critical Care Medicine, Samsung Medical Center, Sungkyunkwan University School of Medicine, Seoul, Korea

Background: Percutaneous dilatational tracheostomy (PDT) performed by an intensivist in critically ill patients is currently popular. Many studies support the safety and feasibility of PDT. However, there is limited data on the safety and feasibility of PDT performed by intensive care trainees.

Methods: To evaluate the safety and feasibility of PDT performed by intensive care trainees and to compare these with those performed by intensivists, we retrospectively analyzed the clinical characteristics and adverse events of all prospectively registered patients who underwent PDT by ICT or intensivists in intensive care units (ICUs) from August 2010 to August 2013.

Results: In the study period, 203 patients underwent PDT in ICUs; 139 (68\%) by trainees and 64 (32\%) by intensivists. There were no statistically significant differences in clinical characteristics including demographics, laboratory findings, and parameters of mechanical ventilation between the two groups. Procedure times and outcomes of the patients were not different between the two groups. The majority of complications observed in 24 hours after PDT were bleeding; however, there was no significant difference between the two groups (trainee $10.8 \%$ vs. intensivist $9.4 \%, \mathrm{p}=0.758$ ). There was no procedure-related death in the two groups.

Conclusions: PDT performed by intensive care trainees was safe and feasible. However, further well-designed studies should be conducted to confirm our results.

Key Words: education; fellowship; intensive care units; surgical procedures, minimally invasive; tracheostomy.

\section{INTRODUCTION}

Tracheostomy is one of the most commonly performed surgical procedures in critically ill patients requiring long term mechanical ventilation or treatment of airway obstruction.[1] Traditionally, this procedure has been performed using surgical principles in operating room. After Sheldon et al.[2] described the Seldinger method of percutaneous tracheostomy placement in 1955, Toye and Weinstein[3] reported a modified percuta-

Received on December 31, 2013

Revised on January 25, 2014

Accepted on February 10, 2014

Correspondence to: Kyeongman Jeon, Department of Critical Care Medicine, Samsung Medical Center, Sungkyunkwan University School of Medicine, 81 Irwon-ro, Gangnam-gu, Seoul 135-710, Korea

Tel: +82-2-3410-2423, Fax:+82-2-2148-7088

E-mail: kjeon@skku.edu neous tracheostomy technique based on Seldinger's principle in 1969. However, the procedure was not used widely due to high risk of complications until Ciaglia et al.[4] introduced percutaneous dilatational tracheostomy (PDT) in 1985. This is also a modified Seldinger technique but offers a variety of advantages, including a small incision and minimized damage in the subcutaneous tissues and easy performance.[4] Since then, the use of PDT has greatly increased because physicians found it a feasible and safe procedure for critically ill patients, replacing conventional surgical tracheostomy.[5]

Recently the subspecialty training program has been established in the field of critical care medicine (CCM) in Korea. However, most of the CCM trainees have non-surgical specialty. Even surgeons-in-training may not have enough experience performing surgical tracheostomy during the training period. PDT is known to be easy to master and safe to perform for trainees

cc This is an Open Access article distributed under the terms of the Creative Commons Attribution Non-Commercial License (http://creativecommons.org/ licenses/by-nc/3.0/) which permits unrestricted non-commercial use, distribution, and reproduction in any medium, provided the original work is properly cited.

(c) 2014 Korean Society of Critical Care Medicine 
without much surgical experience.[6-9] However, the previous reports were focused on PDTs performed by experienced intensivists or PDTs done under the supervision of surgeons.[9-11] Moreover, in Korea, no studies have investigated whether PDT is safe and feasible for subspecialty trainees in CCM. This study aimed to evaluate safety and feasibility of PDT performed by trainees subspecializing in CCM by comparing clinical outcomes and surgical complications between trainee-led PDT and intensivist-led PDT.

\section{MATERIALS AND METHODS}

This is a retrospective observational study conducted with patients who underwent PDT for respiratory support and airway protection at Samsung Seoul Medical Center between August 2011 and July 2013. To assess safety and feasibility of PDT performed by subspecialty trainees in CCM, postprocedural outcomes were compared between trainee-led and intensivist-led procedures.

\section{1) PDT}

The need for PDT was determined after discussion among an intensivist, a subspecialty trainee and an attending physician. In this study, PDTs were performed in the same manner as described in a study by Yoo et al.[9] without the supervision of surgical intensivist. PDT was performed using Ciaglia Blue Rhino ${ }^{\circledR}$ (Cook Medical Inc., Bloomington, IN, USA). As described by Ciaglia et al.[12], after patients were kept in supine position with hyperextension of the neck, the local anesthesia was administered with $2 \%$ lidocaine and a 1 to $1.5 \mathrm{~cm}$ transverse or vertical incision was made at the inferior edge of the cricoid cartilage. The pretracheal soft tissues were bluntly dissected with a mosquito clamp if needed. The underlying trachea was then identified using a needle and introducer sheath under bronchoscopic visualization. After a J-tipped Seldinger wire was inserted through the introducer sheath into the trachea, the introducer sheath was then removed and dilation of the trachea and soft tissue was performed initially with a short dilator fol- lowed by a curved, conical dilator. Finally, the tracheostomy tube was loaded on to an introducer dilator and gently inserted into the trachea over the guidewire through the dilated stoma and secured in place under bronchoscopic visualization. All procedures were performed by either intensivist or subspecialty trainees in CCM at bedside in the ICU. In this study, three intensivists ( 2 medical intensivists and 1 surgical intensivist) had performed PDT from the beginning of PDT introduction in our hospital.[9] The trainees were required to perform at least three PDTs successfully under the supervision of intensivist in addition to participating in three actual operations and attending a training program showing video simulation. CCM trainees include medical doctors $(\mathrm{n}=8)$, neurological doctor $(\mathrm{n}=1)$ and surgical doctor $(\mathrm{n}=1)$.

\section{2) Data collection}

Over the study period, all consecutive patients who underwent PDT in ICU were prospectively registered. The following information was collected on each registered patient: demographic data, underlying conditions, laboratory data, reasons for tracheostomy, data on PDT including timing and duration of the procedure, vital signs monitored during the procedure, and complications developed during the procedure or within 24 hours after the procedure. The procedure time was defined as the time from the sterilization to the connection of the tracheostomy tube with the mechanical ventilator after successful PDT. Postprocedural bleeding was classified into two categories: minor bleeding defined as bleeding from the incision site that required dressing more than three times a day or epinephrine for local hemostasis and major bleeding defined as bleeding that required cauterization, surgical treatment or additional blood transfusion. Cuff perforation and accidental extubation were considered as procedure related complications when occurred within 24 hours after the procedure. Hypoxemia was referred to the episode where pulse oximetry arterial oxygen saturation $\left(\mathrm{SpO}_{2}\right)$ was less than $90 \%$. Arrhythmia developed during the procedure included bradycardia, supraventricular tachycardia (SVT), arterial fibrillation, ventricular tachycardia and ventricular fibrillation (Table 1).

Table 1. Definition of complications

\begin{tabular}{ll}
\hline \hline Complication & \\
\hline Bleeding & Requires multiple dressing change, topical epinephrine, transfusion, or surgical intervention in first 24 h \\
Cuff perforation & Perforation of tracheostomy tube balloon in first $24 \mathrm{~h}$ \\
Accidental decannulation & Unexpected removal of tracheostomy tube in first $24 \mathrm{~h}$ \\
Surgical conversion & Requires surgical tracheostomy by surgical team \\
Hypoxemia & Procedure-associated pulse oximetry arterial oxygen saturation lower than $90 \%$ \\
Arrhythmia & Bradycardia, supraventricular tachycardia during procedure, arterial fibrillation, ventricular tachycardia, and \\
& ventricular fibrillation \\
\hline
\end{tabular}




\section{3) Statistical analysis}

The data are presented as medians and interquartile ranges (IQR) for continuous variables and as numbers and percentages for categorical variables. Data were compared using the MannWhitney U-test for continuous variables and the Chi-square or Fisher's exact test for categorical variables. All tests were two-sided, and a $\mathrm{p}$ value of $<0.05$ was considered significant. The data were analyzed using PASW Statistics 18 (SPSS Inc, Chicago, Illinois).

\section{RESULTS}

A total of 233 patients underwent PDT in the ICU during the study period, and data of 203 patients were analyzed after excluding of 30 patients (13\%) due to insufficient data. Subspecialty trainees in CCM performed PDT in 139 patients (68\%) (trainee group), and intensivists performed PDT in 64 patients (32\%) (intensivist group). There were no differences between the two groups in age, sex, baseline clinical characteristics and blood test results. A PDT was undertaken for one of two reasons: a) to support prolonged mechanical ventilation and b) to secure the airway. There were no significant differences in the reason for PDT between the two groups $(\mathrm{p}=0.189)$. The median duration of mechanical ventilation before tracheostomy was 9 days (5-13) $(p=0.735)$. The setting of ventilation showed no difference between two groups (Table 2).

Table 2. Data of patients underwent percutaneous dilatational tracheostomy in mixed intensive care unit

\begin{tabular}{|c|c|c|c|}
\hline & Trainee group $(n=139)$ & Intensivist group $(\mathrm{n}=64)$ & $\mathrm{p}$ value \\
\hline Age, yr & $67(55-74)$ & $69.5(52-76)$ & 0.797 \\
\hline Males, n (\%) & $95(68)$ & $45(70)$ & 0.778 \\
\hline BMI, $\mathrm{kg} / \mathrm{m}^{2}$ & $21(18-24)$ & $21.5(19-24)$ & 0.578 \\
\hline Platelet, $10^{3} / \mu 1$ & $111(49-221)$ & $128(54-276)$ & 0.274 \\
\hline PT (INR) & $1.16(1.07-1.25)$ & $1.17(1.07-1.29)$ & 0.634 \\
\hline aPTT, sec & $40.1(34.6-46.1)$ & $43.2(36.3-50.6)$ & 0.069 \\
\hline Fibrinogen, & $382(280-559)$ & $393(236-533)$ & 0.445 \\
\hline Underlying conditions & & & 0.089 \\
\hline Hematologic malignancy & $40(28.8)$ & $10(15.4)$ & \\
\hline Oncologic malignancy & $39(28.1)$ & $21(33.8)$ & \\
\hline Pulmonary disease $^{*}$ & $28(20.1)$ & $17(26.2)$ & \\
\hline Chronic kidney disease & $3(2.2)$ & $3(4.6)$ & \\
\hline Trauma & $0(0)$ & $2(3.1)$ & \\
\hline others $^{\dagger}$ & $29(20.9)$ & $12(18.5)$ & \\
\hline Reason for tracheostomy & & & 0.189 \\
\hline Chronic ventilator dependence & $107(77.0)$ & $43(68.3)$ & \\
\hline Airway protection & $32(23.0)$ & $20(31.7)$ & \\
\hline SAPS3 & $65(52.5-78)$ & $58(50-70)$ & 0.056 \\
\hline Period of intubation, $\mathrm{d}$ & $9(5-13)$ & $9(5-13)$ & 0.735 \\
\hline $\mathrm{FiO}_{2},(\%)$ & $60(42.5-80)$ & $60(45-100)$ & 0.404 \\
\hline PEEP, $\mathrm{cmH}_{2} \mathrm{O}$ & $8(8-12)$ & $10(8-12)$ & 0.166 \\
\hline
\end{tabular}

Values expressed as number of patients (\%) or median with interquartile range. ${ }^{*}$ Interstitial lung disease, asthma, chronic obstructive pulmonary disease, tuberculosis, nontuberculous mycobacteria. ${ }^{\dagger}$ Ischemic heart disease, diabetes mellitus, cerebrovascular disease, Parkinson's disease, spinal never injury, aortic dissection. BMI: Body mass index; PT: Prothrombin time; aPTT: activated partial thrombin time; SAPS: Simplified acute physiology score; $\mathrm{FiO}_{2}$ : fraction of inspired oxygen; PEEP: positive end-expiratory pressure.

Table 3. Comparison of procedure times and complications between trainee fellow and staff intensivist

\begin{tabular}{lccc}
\hline \hline & Trainee group $(\mathrm{n}=139)$ & Intensivist group $(\mathrm{n}=64)$ & $\mathrm{p}$ value \\
\hline Procedure time (min), median (IQR) & $22(15-30)$ & $23(15-30)$ & 0.323 \\
Complications & $23(16.5)$ & $9(14.1)$ & 0.652 \\
$\quad$ Bleeding & $15(10.8)$ & $6(9.4)$ & 0.758 \\
$\quad 3$ Major bleeding & 12 & 2 & \\
$\quad$ Minor bleeding & $4(2.9)$ & $0(0)$ & 0.310 \\
Cuff perforation & $2(1.4)$ & $1(1.6)$ & $>0.999$ \\
Hypoxia & $1(0.7)$ & $0(0)$ & $>0.999$ \\
Accidental decannulation & $1(0.7)$ & $1(1.6)$ & 0.532 \\
Arrhythmia & $0(0)$ & $1(1.6)$ & 0.315 \\
Surgical conversion & & & \\
\hline
\end{tabular}

Values expressed as number of patients (\%) or median with interquartile range (IQR). 
Table 4. Comparison of procedure times and complications according to trainee's subspecialty

\begin{tabular}{lccc}
\hline \hline & Medical trainee $(\mathrm{n}=122)$ & Surgical trainee $(\mathrm{n}=17)$ & $\mathrm{p}$ value \\
\hline Procedure time (min), median (IQR) & $23.5(15-30)$ & $20(14-32.5)$ & 0.698 \\
Complications & $21(17.2)$ & $2(11.8)$ & 0.738 \\
Bleeding & $15(12.3)$ & $0(0)$ & 0.215 \\
$\quad$ Major bleeding & $3(2.5)$ & $0(0)$ & \\
Minor bleeding & $12(9.8)$ & $0(0)$ & $>0.999$ \\
Cuff perforation & $4(3.3)$ & $1(0.8)$ & 0.230 \\
Hypoxia & $1(0.8)$ & $0(0)$ & $>0.999$ \\
Accidental decannulation & $1(0.8)$ & $1(0.8)$ & 0.122 \\
Arrhythmia & $0(0)$ & &
\end{tabular}

Values expressed as number of patients (\%) or median with interquartile range (IQR).

Table 5. Outcomes of patients underwent percutaneous dilatational tracheostomy

\begin{tabular}{lccc}
\hline \hline & Trainee group $(\mathrm{n}=139)$ & Intensivist group $(\mathrm{n}=64)$ & $\mathrm{p}$ value \\
\hline Decannulation & $10(7.2)$ & $5(7.8)$ & $>0.999$ \\
Days before decannluation & $45(21-88)$ & $21(19-54)$ & 0.326 \\
Length of ICU stay & $22(11-31)$ & $22(13-36)$ & 0.330 \\
Length of hospital stay & $45(29-80)$ & $44(30-82)$ & 0.836 \\
In hospital death & $42(30.2)$ & $18(28.1)$ & 0.762 \\
\hline
\end{tabular}

Values expressed as number of patients (\%) or median with interquartile range. ICU: intensive care unit.

The procedure time was 22 minutes (15-30) in the trainee group and 23 minutes (15-30) in the intensivist group, showing no significant difference $(\mathrm{p}=0.323)$ (Table 3). PDT-related complications occurred in 23 patients (17\%) in the trainee group and $9(14 \%)$ in the intensivist group $(\mathrm{p}=0.309)$. Bleeding, which was the most frequent complication, occurred in 15 patients $(11 \%)$ in the trainee group and $6(10 \%)$ in the intensivist group, respectively. Other complications did not show significant differences between the two groups (Table 3). Only one of total 203 patients was subject to the switch to surgical tracheostomy due to major bleeding, which occurred during PDT in the intensivist group (Table 4).

Before hospital discharge, decannulation was performed in 10 patients $(7 \%)$ in the trainee group and $5(8 \%)$ in the intensivist group, respectively (Table 5). The median duration from tracheostomy to decannulation was 45 days (21-88) in the trainee group and 21 days (19-54) in the intensivist group, respectively $(\mathrm{p}=0.326)$. The median length of ICU stay was 22 days (11-31) and 22 days (13-36), respectively $(\mathrm{p}=0.330)$ and the median length of hospital stay was 45 days (29-80) and 44 days $(30-82)$, respectively $(p=0.836)$. The number of patients who died due to progression of underlying disease was 42 patients $(30 \%)$ in the trainee group and $18(28 \%)$ in the intensivist group, respectively. However, there was no PDT-related death in both groups.

\section{DISCUSSION}

The purpose of this study was to evaluate safety and feasibility of PDT performed by trainees subspecializing in CCM by comparing clinical outcomes and complications between trainee-led PDT and intensivist-led PDT. This study found no differences in clinical outcomes and procedural complications between the two groups.

It is well documented that PDT is safer and more feasible than conventional surgical tracheostomy.[5,8,9,13,14] PDT is undertaken through approximately $1 \mathrm{~cm}$ incision based on Seldinger technique using bronchoscopy. Two operators are required for the procedure, and one of them identifies a puncture site through the monitor. Because PDT is feasible to perform without the help of surgeons, intensivists perform the procedure at bedside of ICU.[9,15] Although the definition of procedure time required for PDT varies among studies, procedure time for PDT in other studies is shorter, compared with surgical tracheostomy. $[9,15]$ The median procedure time in this study was similar to that of the previous studies.[7] Procedure time between the trainee and intensivist groups was also similar in this study, consistent with a previous report by Johnson et al.[16] that demonstrated no significant difference in procedure time between intensivist and trainee groups.

PDT-related complications rate was similar to the finding of the previous studies $(10 \%-28 \%)$. $[9,13,17,18]$ The rate of complications and complication types between the two groups showed 
The Korean Journal of Critical Care Medicine: Vol. 29, No. 2, May 2014

Table 6. Key outcomes of percutaneous dilatational tracheostomy in the randomized studies comparing percutaneous dilatational and surgical tracheostomy in critically ill patients

\begin{tabular}{lcccccc}
\hline \hline Study & PDT performed by & $\begin{array}{c}\text { No. of patients } \\
\text { performed PDT }\end{array}$ & $\begin{array}{c}\text { Complication } \\
(\mathrm{n})\end{array}$ & $\begin{array}{c}\text { Bleeding } \\
(\mathrm{n})\end{array}$ & $\begin{array}{c}\text { Procedure times, } \\
(\mathrm{min})\end{array}$ & $\begin{array}{c}\text { Use of bronchoscopic } \\
\text { guidedance for PDT }\end{array}$ \\
\hline Silvester et al.[15] & Trainee & 100 & NA & 7 & 20 (IQR, 15-30) & Yes \\
Hazard et al.[19] & Trainee & 24 & 3 & 1 & NA & No \\
Tabaee et al.[20] & Trainee & 29 & 7 & 1 & 8 (range, 3-25) & Yes \\
Massick et al.[21] & Trainee & 50 & 4 & 2 & 11 (IQR, 4-18) & Yes \\
Wu et al.[10] & Staff & 42 & NA & 2 & $22 \pm 12$ & Yes \\
Heikkinen et al.[14] & Staff & 31 & NA & 5 & $35 \pm 12$ & No \\
Antonelli et al.[22] & Staff & 67 & 10 & 2 & $17 \pm 10$ & No \\
Melloni et al.[23] & Staff & 25 & 2 & 2 & $14 \pm 6$ & Yes \\
\hline
\end{tabular}

PDT: percutaneous dilatational tracheostomy; NA: not available; IQR: interquartile range; staff: intensive care specialists; trainee: doctor in resident program or critical care fellowship program.

no significant differences in this study. Previous studies also found no significant differences in the procedure related complications in trainee-led PDT group (Table 6).[10,14,15,19-23] Bleeding occurred in $5 \%$ to $24 \%$ of patients who underwent PDT.[7,9,16,24] This study also found a similar incidence rate of bleeding between the trainee and intensivist groups. In addition, all PDT procedures performed during the study period did not require the supervision of surgeons, although Suresh [25] suggested the need for surgical expertise to perform PDT. Minor bleeding was controlled without a surgical treatment, and major bleeding was controlled by intensivists or trainees with surgical background. Unlike Suresh,[25] we suggest that surgeons or the supervision of surgeons is not definitely required for PDT but underline the readiness to perform emergency surgical hemostasis, when necessary. Some complications including failed tracheal puncture, esophageal rupture, pneumothorax and paratracheal insertion of tracheostomy tube, were previously reported $[5,13]$ but have not been identified in this study. These complications tend to occur when PDT is undertaken without bronchoscopic assistance.[26] Because PDT was completed with bronchoscopy, such complications could be prevented in this study.

This study had several limitations, including a retrospective study design and small sample size. First, this study was conducted at a tertiary hospital with multidisciplinary care. Therefore the results of this study cannot be generalized to other hospitals that have a subspecialty training program but not provide multidisciplinary care. Second, the likelihood of bias cannot be fully ruled out because there could be undocumented inclusion criteria for subspecialty trainees participated in PDT procedure although we described their work schedule as a key factor deciding who would perform PDT. Also, effects of PDTs performed by intensivists on the results of this study appear to be trivial given that most of their procedures were undertaken for educa- tional purpose. Lastly, while early complications associated with PDT were identified, other complications such as infection of surgical site could not be evaluated through the medical records. It was also impossible to identify post-extubation complications such as tracheal stenosis with a small number of patients who underwent decannulation.

In conclusion, no differences were found between trainee-led PDT and intensivist-led PDT with respect to clinical outcomes and complications. We suggest that PDT is a safe and feasible procedure to be performed by subspecialty trainees in CCM at institutions providing multidisciplinary medical practices that they are well prepared for an emergency.

\section{REFERENCES}

1) Heffner JE: Timing of tracheotomy in mechanically ventilated patients. Am Rev Respir Dis 1993; 147: 768-71.

2) Shelden CH, Pudenz RH, Freshwater DB, Crue BL: A new method for tracheotomy. J Neurosurg 1955; 12: 428-31.

3) Toy FJ, Weinstein JD: A percutaneous tracheostomy device. Surgery 1969; 65: 384-9.

4) Ciaglia P, Firsching R, Syniec C: Elective percutaneous dilatational tracheostomy. A new simple bedside procedure; preliminary report. Chest 1985; 87: 715-9.

5) Cabrini L, Monti G, Landoni G, Biondi-Zoccai G, Boroli F, Mamo D, et al: Percutaneous tracheostomy, a systematic review. Acta Anaesthesiol Scand 2012; 56: 270-81.

6) Petros S, Engelmann L: Percutaneous dilatational tracheostomy in a medical ICU. Intensive Care Med 1997; 23: 630-4.

7) Beiderlinden M, Karl Walz M, Sander A, Groeben H, Peters $\mathrm{J}$ : Complications of bronchoscopically guided percutaneous dilational tracheostomy: beyond the learning curve. Intensive Care Med 2002; 28: 59-62. 
8) Díaz-Regañón G, Miñambres E, Ruiz A, González-Herrera S, Holanda-Peña M, López-Espadas F: Safety and complications of percutaneous tracheostomy in a cohort of 800 mixed ICU patients. Anaesthesia 2008; 63: 1198-203.

9) Yoo H, Lim SY, Park CM, Suh GY, Jeon K: Safety and Feasibility of Percutaneous Tracheostomy Performed by Medical Intensivists. Korean J Crit Care Med 2011; 26: 261-6.

10) Wu JJ, Huang MS, Tang GJ, Shih SC, Yang CC, Kao WF, et al: Percutaneous dilatational tracheostomy versus open tracheostomy--a prospective, randomized, controlled trial. J Chin Med Assoc 2003; 66: 467-73.

11) Freeman BD, Isabella K, Cobb JP, Boyle WA 3rd, Schmieg $\mathrm{RE} \mathrm{Jr}$, Kolleff $\mathrm{MH}$, et al: A prospective, randomized study comparing percutaneous with surgical tracheostomy in critically ill patients. Crit Care Med 2001; 29: 926-30.

12) Ciaglia P: Percutaneous tracheostomy. Crit Care Med 1992; 20: 904

13) Freeman BD, Isabella K, Lin N, Buchman TG: A meta-analysis of prospective trials comparing percutaneous and surgical tracheostomy in critically ill patients. Chest 2000; 118: 1412-8.

14) Heikkinen M, Aarnio P, Hannukainen J: Percutaneous dilational tracheostomy or conventional surgical tracheostomy? Crit Care Med 2000; 28: 1399-402.

15) Silvester W, Goldsmith D, Uchino S, Bellomo R, Knight S, Seevanayagam S, et al: Percutaneous versus surgical tracheostomy: A randomized controlled study with long-term follow-up. Crit Care Med 2006; 34: 2145-52.

16) Johnson JL, Cheatham ML, Sagraves SG, Block EF, Nelson LD: Percutaneous dilational tracheostomy: a comparison of single- versus multiple-dilator techniques. Crit Care Med 2001; 29: 1251-4.

17) Añón JM1, Gómez V, Escuela MP, De Paz V, Solana LF, De La Casa RM, et al: Percutaneous tracheostomy: comparison of Ciaglia and Griggs techniques. Crit Care 2000; 4: 124-8.
18) Walz MK, Peitgen K, Thürauf N, Trost HA, Wolfhard U, Sander A, et al: Percutaneous dilatational tracheostomy-early results and long-term outcome of 326 critically ill patients. Intensive Care Med 1998; 24: 685-90.

19) Hazard P, Jones C, Benitone J: Comparative clinical trial of standard operative tracheostomy with percutaneous tracheostomy. Crit Care Med 1991; 19: 1018-24.

20) Tabaee A, Geng E, Lin J, Kakoullis S, McDonald B, Rodriguez $\mathrm{H}$, et al: Impact of neck length on the safety of percutaneous and surgical tracheotomy: a prospective, randomized study. Laryngoscope 2005; 115: 1685-90.

21) Massick DD, Yao S, Powell DM, Griesen D, Hobgood T, Allen JN, et al: Bedside tracheostomy in the intensive care unit: a prospective randomized trial comparing open surgical tracheostomy with endoscopically guided percutaneous dilational tracheotomy. Laryngoscope 2001; 111: 494-500.

22) Antonelli M, Michetti V, Di Palma A, Conti G, Pennisi MA, Arcangeli A, et al: Percutaneous translaryngeal versus surgical tracheostomy: A randomized trial with 1-yr double-blind follow-up. Crit Care Med 2005; 33: 1015-20.

23) Melloni G, Muttini S, Gallioli G, Carretta A, Cozzi S, Gemma M, et al: Surgical tracheostomy versus percutaneous dilatational tracheostomy. A prospective-randomized study with long-term follow-up. J Cardiovasc Surg (Torino) 2002; 43: 113-21.

24) Delaney A, Bagshaw SM, Nalos M: Percutaneous dilatational tracheostomy versus surgical tracheostomy in critically ill patients: a systematic review and meta-analysis. Crit Care 2006; 10: R55.

25) Suresh D: Percutaneous dilational tracheostomy--a caution. Anaesth Intensive Care 1990; 18: 425-6.

26) Mansharamani NG, Koziel H, Garland R, LoCicero J 3rd, Critchlow J, Ernst A: Safety of bedside percutaneous dilatational tracheostomy in obese patients in the ICU. Chest 2000; 117: 1426-9. 